\title{
Evaluation of Plaque Stabilization by Fluvastatin with Carotid Intima- Medial Elasticity Measured by a Transcutaneous Ultrasonic-Based Tissue Characterization System
}

\author{
Toshio Yamagishi ${ }^{1}$, Makoto Kato ${ }^{2}$, Yoshiro Koiwa ${ }^{3}$, Ken Omata ${ }^{4}$, Hideyuki Hasegawa ${ }^{5,6}$, and Hiroshi Kanai ${ }^{6,5}$ \\ ${ }^{1}$ Department of Internal Medicine, Tohoku Kosai Hospital, Sendai, Japan \\ ${ }^{2}$ Panasonic Shikoku Electronics Co., Ltd, Yokohama, Japan \\ ${ }^{3}$ Oizumi Memorial Hospital, Shiroishi, Japan \\ ${ }^{4}$ Health Administration Center, Miyagi University of Education, Sendai, Japan \\ ${ }^{5}$ Department of Biomedical Engineering, Graduate School of Biomedical Engineering, Tohoku University, Sendai, Japan \\ ${ }^{6}$ Department of Electronic Engineering, Graduate School of Engineering, Tohoku University, Sendai, Japan
}

\begin{abstract}
Aim: As an approach to tissue characterization, we attempted to classify in vivo carotid plaque tissues in terms of arterial wall elasticity instead of echogenicity on B-mode scanning and investigated whether the effect of fluvastatin on carotid elasticity can be detected in hypercholesterolemic patients. Methods: In 170 subjects, simultaneous measurements of intima-media thickness (IMT) and elastic modulus in the circumferential direction $\left(E_{\theta}\right)$ were performed using a new transcutaneous high-resolution Doppler technique.

Results: From the observation of various tissues, an elasticity library was obtained as follows: lipid core, $22 \pm 15 \mathrm{kPa}$; calcification, $674 \pm 384 \mathrm{kPa}$; lipid core- and calcification-free plaques, $173 \pm 69 \mathrm{kPa}$; smooth muscle, $104 \pm 32 \mathrm{kPa}$; blood clot, $85 \pm 68 \mathrm{kPa}$; fibrosis, $273 \pm 173 \mathrm{kPa}$.

The effect of fluvastatin $(30 \mathrm{mg} / \mathrm{day}, n=62)$ was assessed over 12 months using the elasticity distribution and serum markers. The statin reduced low-density lipoprotein cholesterol, high-sensitivity $\mathrm{CRP}$, mean IMT and mean $E_{\theta}$, and increased nitrite/nitrate. In the max IMT $\geq 1.1 \mathrm{~mm}$ group, both $E_{\theta}$ and IMT decreased significantly. On the other hand, in the max IMT $<1.1 \mathrm{~mm}$ group, $E_{\theta}$ but not IMT decreased significantly. The histogram of the subgroups showing increased $E_{\theta}$ with max IMT $\geq 1.1 \mathrm{~mm}$ revealed a decrease in areas corresponding to $E_{\theta}$ of $20-200 \mathrm{kPa}$ (lipid/smooth muscle-rich tissue) and an increase in relatively hardened areas of $<250 \mathrm{kPa}$ (collagen fibers).

Conclusion: Non-invasive echographic carotid arterial elasticity measurement is useful for the classification of atherosclerotic plaques and evaluation of chronological and histopathological changes.
\end{abstract}

J Atheroscler Thromb, 2009; 16:662-673.

Key words; Elasticity, Statin, Echo, Carotid artery

\section{Introduction}

Ultrasonography of the carotid arteries is a common imaging study performed for the diagnosis of atherosclerosis, not only in carotid but also in systemic arteries. Conventional evaluations are based on the

Address for correspondence: Toshio Yamagishi, Department of Internal Medicine, Tohoku Kosai Hospital, 2-3-11, Kokubuncho, Aoba-ku, Sendai 980-0803, Japan

E-mail: tyamagishi@tohokukosai.com

Received: January 8, 2009

Accepted for publication: April 16, 2009 measurement of carotid arterial intima-media thickness (IMT) to detect morphological changes and remodeling, such as thickening and calcification ${ }^{1-5)}$ and on integrated backscatter (IBS) analysis using echogenicity on B-mode scanning for the soft/hard characterization of plaque composition ${ }^{6-8)}$; however, these procedures provide no information on the mechanical stability of plaque against rupture and for tissue characterization. Recently, a new ultrasonic system that can measure regional elasticity in the arterial wall utilizing the phased tracking method has been developed ${ }^{9-15}$. Measurements of the elasticity distribu- 
Table 1. Characteristics of the study population

\begin{tabular}{lccc}
\hline & $\begin{array}{c}\text { Healthy } \\
\text { volunteers in } \\
20 s-40 s\end{array}$ & $\begin{array}{c}\text { Patients with } \\
\text { hyperlipidemia }\end{array}$ & p-value \\
& 46 & 72 & \\
\hline $\mathrm{N}$ & $35.4 \pm 2.7$ & $60.4 \pm 7.6$ & $<0.0001$ \\
age & $0.54 \pm 0.08$ & $0.88 \pm 0.25$ & $<0.0001$ \\
mean IMT $(\mathrm{mm})$ & $0.68 \pm 0.08$ & $1.15 \pm 0.20$ & $<0.0001$ \\
max IMT $(\mathrm{mm})$ & $118.8 \pm 26.8$ & $158.5 \pm 62.0$ & 0.0006 \\
mean $E_{\theta}(\mathrm{kPa})$ & $111.2 \pm 7.8$ & $135.2 \pm 13.8$ & $<0.0001$ \\
SBP $(\mathrm{mmHg})$ & $70.1 \pm 7.4$ & $81.3 \pm 8.5$ & $<0.0001$ \\
DBP $(\mathrm{mmHg})$ & $41.1 \pm 9.0$ & $54.0 \pm 11.7$ & 0.0001 \\
PP $(\mathrm{mmHg})$ & $70.8 \pm 8.9$ & $71.3 \pm 9.6$ & $\mathrm{NS}$ \\
PR $(\mathrm{bpm})$ & $177.6 \pm 45.5$ & $255.4 \pm 32.8$ & $<0.0001$ \\
TC $(\mathrm{mg} / \mathrm{dL})$ & $119.2 \pm 70.7$ & $141.9 \pm 96.1$ & $\mathrm{NS}$ \\
TG $(\mathrm{mg} / \mathrm{dL})$ & $50.7 \pm 10.3$ & $59.8 \pm 15.9$ & $\mathrm{NS}$ \\
$\mathrm{HDL}-\mathrm{C}(\mathrm{mg} / \mathrm{dL})$ & $98.1 \pm 29.8$ & $154.7 \pm 21.8$ & $<0.0001$ \\
$\mathrm{LDL}-\mathrm{C}(\mathrm{mg} / \mathrm{dL})$ & $87.0 \pm 9.3$ & $98.8 \pm 13.1$ & 0.0128 \\
FPG $(\mathrm{mg} / \mathrm{dL})$ & $4.7 \pm 0.5$ & $5.2 \pm 0.7$ & 0.0222 \\
HbAlc $(\%)$ & $1,106.4 \pm 220.4$ & $1,592.0 \pm 306.9$ & $<0.0001$ \\
baPWV $(\mathrm{cm} / \mathrm{s})$ & & &
\end{tabular}

SBP: systolic blood pressure, DBP: diastolic blood pressure, PP: pulse pressure, PR: pulse rate, TC: total cholesterol, TG: triglyceride, HDL-C: high density lipoprotein cholesterol, LCL-C: low density lipoprotein cholesterol, FPG: fasting plasma glucose, baPWV: brachio-ankle pulse velocity wave.

tion of lipids, blood clots, fibrous tissue and calcified tissue in vitro have been reported previously, and an elasticity image can be classified into tissue components using the reference data obtained by in vitro experiments ${ }^{16)}$. The usefulness of measuring the elastic modulus of the common carotid arteries (CCAs) by ultrasound imaging to detect early-stage atherosclerotic lesions caused by risk factors with regard to lifestyle-related disease has also been reported ${ }^{15}$.

The aim of the present study was to evaluate and classify in vivo carotid plaque tissues in terms of arterial wall elasticity using this novel real-time ultrasonic measurement system rather than conventional methods. Carotid plaque was evaluated to assess atherosclerotic lesions, such as plaques, that showed an increase of elasticity with progressive lipid deposition and fibrosis resulting from chronological and histopathological changes. In addition, we assessed whether the effects of fluvastatin on plaque stabilization can be detected by carotid elasticity distribution in patients with hypercholesterolemia.

\section{Methods}

\section{Study Population}

A total of 170 subjects, including healthy volun-
Table 2. Characteristics of patients with lipid core or calcification in carotid arteries

\begin{tabular}{lcc}
\hline & $\begin{array}{c}\text { Patients with } \\
\text { lipid core }\end{array}$ & $\begin{array}{c}\text { Patients with } \\
\text { calcification }\end{array}$ \\
\hline $\mathrm{N}$ & 18 & 33 \\
age & $56.8 \pm 9.1$ & $61.8 \pm 8.9$ \\
mean IMT $(\mathrm{mm})$ & $0.91 \pm 0.16$ & $0.93 \pm 0.17$ \\
max IMT $(\mathrm{mm})$ & $1.21 \pm 0.20$ & $1.26 \pm 0.27$ \\
mean $E_{\theta}(\mathrm{kPa})$ & $145.7 \pm 40.6$ & $193.7 \pm 55.4$ \\
$\mathrm{SBP}(\mathrm{mmHg})$ & $129.8 \pm 20.0$ & $140.1 \pm 17.7$ \\
$\mathrm{DBP}(\mathrm{mmHg})$ & $79.8 \pm 14.1$ & $81.2 \pm 9.9$ \\
$\mathrm{PP}(\mathrm{mmHg})$ & $50.0 \pm 13.5$ & $58.9 \pm 12.6$ \\
$\mathrm{PR}(\mathrm{bpm})$ & $71.5 \pm 12.8$ & $72.3 \pm 10.6$ \\
$\mathrm{TC}(\mathrm{mg} / \mathrm{dL})$ & $230.4 \pm 36.8$ & $228.6 \pm 34.5$ \\
$\mathrm{TG}(\mathrm{mg} / \mathrm{dL})$ & $148.5 \pm 82.3$ & $117.5 \pm 52.7$ \\
$\mathrm{HDL}-\mathrm{C}(\mathrm{mg} / \mathrm{dL})$ & $52.6 \pm 13.9$ & $56.4 \pm 13.4$ \\
$\mathrm{LDL}-\mathrm{C}(\mathrm{mg} / \mathrm{dL})$ & $140.5 \pm 37.5$ & $137.0 \pm 30.1$ \\
$\mathrm{FPG}(\mathrm{mg} / \mathrm{dL})$ & $116.9 \pm 45.5$ & $104.1 \pm 24.6$ \\
$\mathrm{HbA}(\%)$ & $5.5 \pm 1.3$ & $5.2 \pm 0.6$ \\
$\mathrm{baPWV}(\mathrm{cm} / \mathrm{s})$ & $1,421.1 \pm 144.4$ & $1,644.4 \pm 306.6$ \\
\hline
\end{tabular}

SBP: systolic blood pressure, DBP: diastolic blood pressure, PP: pulse pressure, PR: pulse rate, TC: total cholesterol, TG: triglyceride, HDL-C: high density lipoprotein cholesterol, LCL-C: low density lipoprotein cholesterol, FPG: fasting plasma glucose, baPWV: brachio-ankle pulse velocity wave.

teers, outpatients with untreated hypercholesterolemia, patients with lipid core or calcification, and a single case of acute-onset carotid artery dissection, were enrolled in this study from 2004 to 2006.

The characteristics of the study population, except the case of carotid artery dissection, are shown in Table 1, 2. Seventy-two subjects with hypercholesterolemia were defined in accordance with the guidelines of the Japanese Society of Atherosclerosis. Hypercholesterolemia was defined as total cholesterol $\geq 220$ $\mathrm{mg} / \mathrm{dL}$ or low-density lipoprotein cholesterol (LDL-C) $\geq 140 \mathrm{mg} / \mathrm{dL}$ and triglyceride (TG) $\geq 150 \mathrm{mg} / \mathrm{dL}$. None of the subjects had hypertension, diabetes, a history of recent cardiovascular events, ischemic heart disease, atrial fibrillation, arteriosclerosis obliterans (ASO, defined as an ankle/brachial blood pressure index $[\mathrm{ABI}]<0.9)$, renal insufficiency with serum creatinine greater than $3 \mathrm{mg} / \mathrm{dL}$, or severe hepatic disorder. Patients with congestive heart failure were excluded from this study.

Some patients with lipid core and/or calcification on B-mode images included untreated patients with hypertension, diabetes mellitus, hypertension being defined as systolic blood pressure (BP) $\geq 140 \mathrm{mmHg}$ and/or diastolic BP $\geq 90 \mathrm{mmHg}$. Diabetes mellitus was defined as a fasting plasma glucose (FPG) $\geq 126$ 
$\mathrm{mg} / \mathrm{dL}$. They did not have any complications.

None of the patients were taking any drugs, such as inhibitors of the renin-angiotensin system, Ca channel blockers and anti-diabetic agents.

Similar examinations were conducted in 46 healthy subjects between 20 and 49 years of age, who served as the control group.

In addition, we assessed the effect of fluvastatin $(30 \mathrm{mg} / \mathrm{day}, n=62)$ during a period of 12 months, evaluating IMT, elasticity distribution, brachial-ankle pulse wave velocity (baPWV) and serum and markers, including high-sensitivity CRP (hs-CRP) and nitrite/ nitrate $(\mathrm{NOx})$. In the control group $(n=10)$, no statin treatment was performed.

The subjects provided informed consent, and the study was approved by the Institutional Ethics Committees of Tohoku Kosai Hospital and Matsushita Electrical Industrial Co., Ltd.

\section{Carotid IMT and Elasticity Measurements}

The subjects were studied after having abstained from caffeine, alcohol, and smoking during the previous 12 hours. They rested in a supine position for 15 $\mathrm{min}$ in a quiet room before the baseline hemodynamic measurements were obtained. Brachial blood pressure and heart rate (HR) (mean of three readings) were measured in the right arm with an automated digital oscillometric sphygmomanometer (EW-3110; Matsushita Electric Works, Osaka, Japan). An imaging study of the bilateral common carotid arteries was performed using a newly developed high-resolution ultrasonic measurement system (Panasonic, prototype) with a center frequency of 7.5 MHz. One well-trained sonographer who regularly participates in quality control measurement sessions performed all ultrasound scans ${ }^{13,15)}$. The sonographer has been trained to obtain an elastic modulus measurement deviation of only $6 \%$ in the same subject (inter- and intra- variations were less than $10 \%$ at our institution). In order to obtain an accurate measurement of the same sites of carotid arteries in repeated measurements, the sonographer performed IMT and elastic modulus measurements by comparing current $\mathrm{B}$-mode images with past B-mode images on the PC display and positioned the probe so as to measure a similar position in the carotid arteries.

IMT can only be determined accurately in the far wall position because only the far wall IMT is defined by leading edges, which enables correct ultrasonographic representation; therefore, the far walls of the following sites were measured: (1) the distal $3 \mathrm{~cm}$ of the straight part of both CCAs and (2) the distal 1 $\mathrm{cm}$ of both carotid bulbs. As in several previous studies, we defined a plaque as a focal raised lesion, using an IMT cutoff value of $1.1 \mathrm{~mm}$. Mean IMT and max IMT were determined to be the mean value of IMT and the maximum value of IMT, respectively, at six locations in the bilateral CCAs, including the carotid bulb.

This system measures the radial strain of the arterial wall during one cardiac cycle and calculates the elasticity from radial strain and pulse pressure. The distribution of elasticity is displayed as a $2 \mathrm{D}$ cross-sectional color-coded image in B-mode imaging, and the image is updated at every heartbeat. To measure minute changes in thickness during one cardiac cycle, this system uses the phased tracking method ${ }^{5-10)}$. Elasticity was estimated at intervals of $80 \mu \mathrm{m}$ along each ultrasonic beam in the depth direction and $400 \mu \mathrm{m}$ in the arterial longitudinal direction. From the maximum change in thickness, $\left(h_{\max }-h_{\min }\right)$, during one heartbeat, the radial strain $\varepsilon_{\mathrm{r}}$ of each assigned layer in the artery wall was calculated as follows:

$$
\varepsilon_{\mathrm{r}}=\left(\mathrm{h}_{\max }-\mathrm{h}_{\min }\right) / \mathrm{h}_{\max },
$$

where $h_{\max }$ and $h_{\min }$ are the maximum and minimum thickness of an assigned layer in the wall during one heartbeat, respectively.

The elastic modulus in the radial direction $(E \mathrm{r})$ and that in the circumferential direction $\left(E_{\theta}\right)$ were calculated as follows:

$$
\begin{aligned}
& E \mathrm{r}=\text { Pulse Pressure } / \varepsilon_{\mathrm{r}} \\
& E_{\theta}=(1 / 2) \times\left(\mathrm{r}_{0} / \mathrm{h}_{0}+1\right) \times E \mathrm{r} .
\end{aligned}
$$

In these formulae, pulse pressure (PP) is the difference between systolic and diastolic brachial blood pressure (carotid blood pressure would be more accurate but is difficult to measure), and ho and $\mathrm{r}_{0}$ are the initial wall thickness and radius of the vessel in end-diastole, respectively. The radial strain $\varepsilon_{\mathrm{r}}$ is caused by radial compression and circumferential stretching due to the increase of blood pressure $\Delta \mathrm{p}$, with longitudinal strain being disregarded. As this circumferential stretching produces radial strain due to the incompressibility of the arterial wall, both radial and circumferential stresses should be considered when evaluating elasticity; however, as shown in Eq. (2), Er is defined as the ratio of pulse pressure (which corresponds to radial stress) and radial strain. The circumferential stress acting on the cylindrical shell is not identical to pulse pressure, but also depends on ro/ho. Thus, $E \mathrm{r}$ is apparent elasticity in the radial direction and is dependent on $\mathrm{r} / \mathrm{h}_{0}$ as well as on the elasticity of the arterial wall. Alternatively, $E_{\theta}$ of Eq. (3) was introduced to evaluate the elasticity of the arterial wall by suppressing the influence of $\mathrm{r} 0 / \mathrm{h}^{7}{ }^{7}$. The elastic modulus in each instrumentation locus was expressed in a histo- 
gram, and the elasticity distribution of each tissue composition was determined.

The length and thickness of the ROI (region of interest), in which the elasticity distribution and average elasticity were calculated, can be changeable and depend on the effective size of the interested area, including IMT. When we obtained the elasticity library, by referring to B-mode echo images, we changed the sizes and positions of ROI within plaques of patients in order to confirm that areas with extreme differences in brightness and/or echo pattern are not included in the region of interest.

\section{PWV Measurements}

Brachial-ankle PWV (baPWV) was measured using a volume-plethysmographic apparatus (Form/ ABI; Colin Co., Ltd., Komaki, Aichi, Japan) by simultaneous BP and waveform measurements on all four limbs, in addition to ECG and phonogram tracings. PWV was automatically calculated by time-phasic analysis using the following formula: distance between two sites divided by pulse wave transit time. Values for baPWV were measured from the ascending point shown by the right brachial pulse volume recorder to the ascending point indicated by each ankle pulse volume recorder. The distance between the right brachium and ankle was estimated as described previously ${ }^{16)}$.

\section{Blood Examination, NOx and hs-CRP}

The patients were requested to fast overnight without medication until blood samples were drawn. Venous blood samples were then drawn for analysis of FPG and serum concentrations of total cholesterol (TC), TG, high-density lipoprotein cholesterol (HDL-C) and HbA1c by standard laboratory methods. Serum LDL-C was calculated by Friedewald's equation. None of the patients had a TG $>400 \mathrm{mg} /$ $\mathrm{dL}$, at which the Friedewald equation is known to yield unreliable results.

The serum level of $\mathrm{NOx}$ (nitrite/nitrate; $\mathrm{NO}_{2}-/$ $\mathrm{NO}_{3-}-$, an indicator of $\mathrm{NO}$, was measured by the Griess reaction after nitrate reductase treatment ${ }^{17)}$ with a flow injection analysis system (Tokyo Chemical Industry Co., Ltd., Tokyo, Japan). The serum NOx level was $10-71 \mu \mathrm{mol} / \mathrm{L}$ and the coefficient of its variation was $3.0 \%$.

The serum level of hs-CRP was measured by the BNII High Sensitivity CRP assay using anti-CRP monoclonal antibody coated with polystyrene particles and fixed-time kinetic nephelometric measurements (N High Sensitivity CRP; Dadebehring Inc., Marburg, Germany) with a lower detection level of 0.2

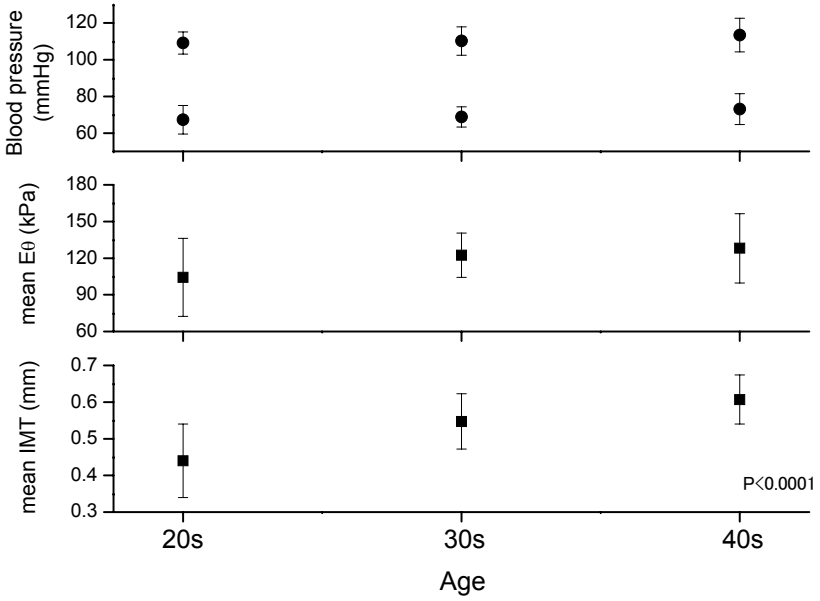

Fig. 1. Effects of aging on IMT and elastic modulus in healthy volunteers.

IMT increased with greater age, but not with higher blood pressure. Overall, $E_{\theta}$ in healthy volunteers was not affected by aging.

$\mathrm{mg} / \mathrm{L}$ and a coefficient of variation of $3.0 \%{ }^{18)}$.

\section{Statistical Analysis}

Data were expressed as the mean \pm SD. Statistical analysis was performed using a $\mathrm{JMP}^{\circledR}$ software package (JMP 7.0.1; SAS Institute, USA), a value of $p<$ 0.05 being considered significant. Differences between groups were analyzed by one-way analysis of variance (ANOVA). A post-hoc test for multiple comparisons was used when ANOVA showed significance. Linear regression analysis was performed to evaluate the association between IMT, elasticity or baPWV, and other clinical variables.

\section{Results}

Table 1 shows the characteristics of the study population of healthy volunteers $(n=46)$, hypercholesteremia patients $(n=72)$, patients with a lipid core in carotid artery plaque $(n=18)$, and patients with calcification $(n=33)$. The average blood pressure (systolic/diastolic) was significantly lower in healthy volunteers than in other subjects (ANOVA, $p<0.0001$ ) and other risk factors for cardiovascular disease were also lower in healthy volunteers.

\section{Elasticity Library}

The effects of aging on the mean IMT and mean $E_{\theta}$ in healthy volunteers in their twenties $(n=15)$, thirties $(n=12)$ and forties $(n=19)$ are shown in Fig. 1. We were unable to obtain data for healthy volunteers over fifty. Although IMT increased signifi- 
A

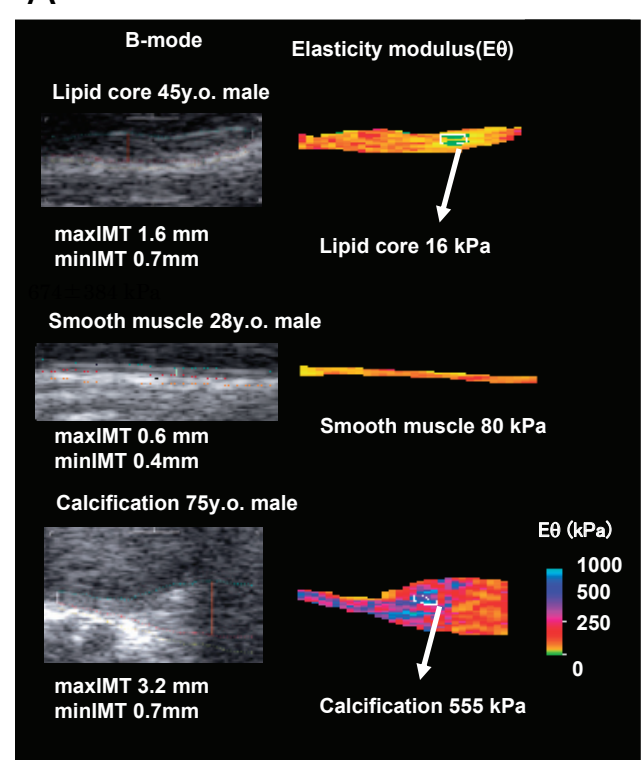

B

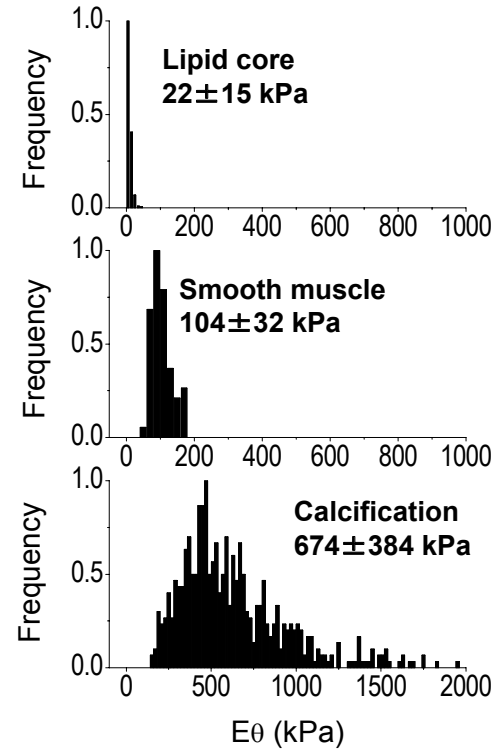

Fig. 2. Elastic modulus of lipid core, smooth muscle and calcification.

The right panel (A) shows the relation between B-mode findings and calculated $E_{\theta}$. The value of $E_{\theta}$ was related to tissue composite elements. The left panel (B) shows a histogram for $E_{\theta}$ (lipid core, smooth muscle and calcification).

cantly by age, there was no significant increase in $E_{\theta}$ or blood pressure. In healthy volunteers in their thirties and forties, no significant increases in $\mathrm{TC}$, HDL-C, LDL-C, TG or FPG were found in comparison with levels in healthy volunteers in their twenties (data not shown).

Fig. 2A shows the relationship between B-mode findings and $E_{\theta}$ in three representative cases. The dark central region of soft plaques was considered to be the lipid core $(16 \mathrm{kPa})$. The normal carotid intima-media complex in a 28-year-old man was considered to be smooth muscle $(80 \mathrm{kPa})$. The white regions, which were not accompanied by an acoustic shadow, were considered to be calcified regions $(555 \mathrm{kPa})$. All data are summarized as histograms in Fig. 2B (lipid core, $22 \pm 15 \mathrm{kPa}$ (lesions: $n=18$ ); calcification, $674 \pm 384$ $\mathrm{kPa}$ (lesions: $n=36)$ ). In the 15 healthy subjects who were free from vascular risk factors, the normal carotid intima-media complex was considered to be smooth muscle $(104 \pm 32 \mathrm{kPa}$, sites: $n=71)$.

Next, in order to obtain the elasticity of blood clots and fibrosis, the following case was used. A 43-year-old woman came to our department because of a pulsatile mass $2.5 \mathrm{~cm}$ in size in the right submandibular area. Her blood pressure was $108 / 80 \mathrm{mmHg}$, and ECG showed sinus tachycardia $(92 / \mathrm{min})$. Imaging of enhanced CT and ultrasound showed acuteonset carotid artery dissection. The patient had no cardiovascular risk and no history of stroke, and had experienced a spontaneous cure from intra-pseudosaccular thrombosis. During B-mode scanning to observe the healing process at our clinic, with permission from the patient and approval from our ethics committee, elastic modulus was measured several times (Fig.3). Elasticity $E_{\theta}$, as determined from the time course of changes, was $85 \pm 68 \mathrm{kPa}$ in areas corresponding to thrombi and $273 \pm 173 \mathrm{kPa}$ in areas of fibrosis.

From the observation of various tissues, the elasticity library of $E_{\theta}$ by transcutaneous ultrasound was obtained, as shown in Fig. 4. The data indicate that the $E_{\theta}$ value rises depending on the tissue constituents. The mean $E_{\theta}$ value of lipid core- and calcification-free plaques was $173 \pm 69 \mathrm{kPa}$ (lesions: $n=270$ ), falling between the $E_{\theta}$ values of fibrosis and blood clot.

\section{Effect of Fluvastatin On Elastic Modulus and IMT}

At baseline, patients with hypercholesterolemia showed significantly higher LDL-C, baPWV, mean IMT, max IMT and mean $E_{\theta}$ than seen in healthy volunteers (Table 1). As shown in Fig. 5, in the control group of hypercholesterolemia $(n=10)$, no change of LDL-C was seen. In the group treated with fluvastatin $(n=62)$, LDL-C was significantly reduced immediately. By fluvastatin treatment, hs-CRP and baPWV decreased gradually but NO increased over time $(p<$ 


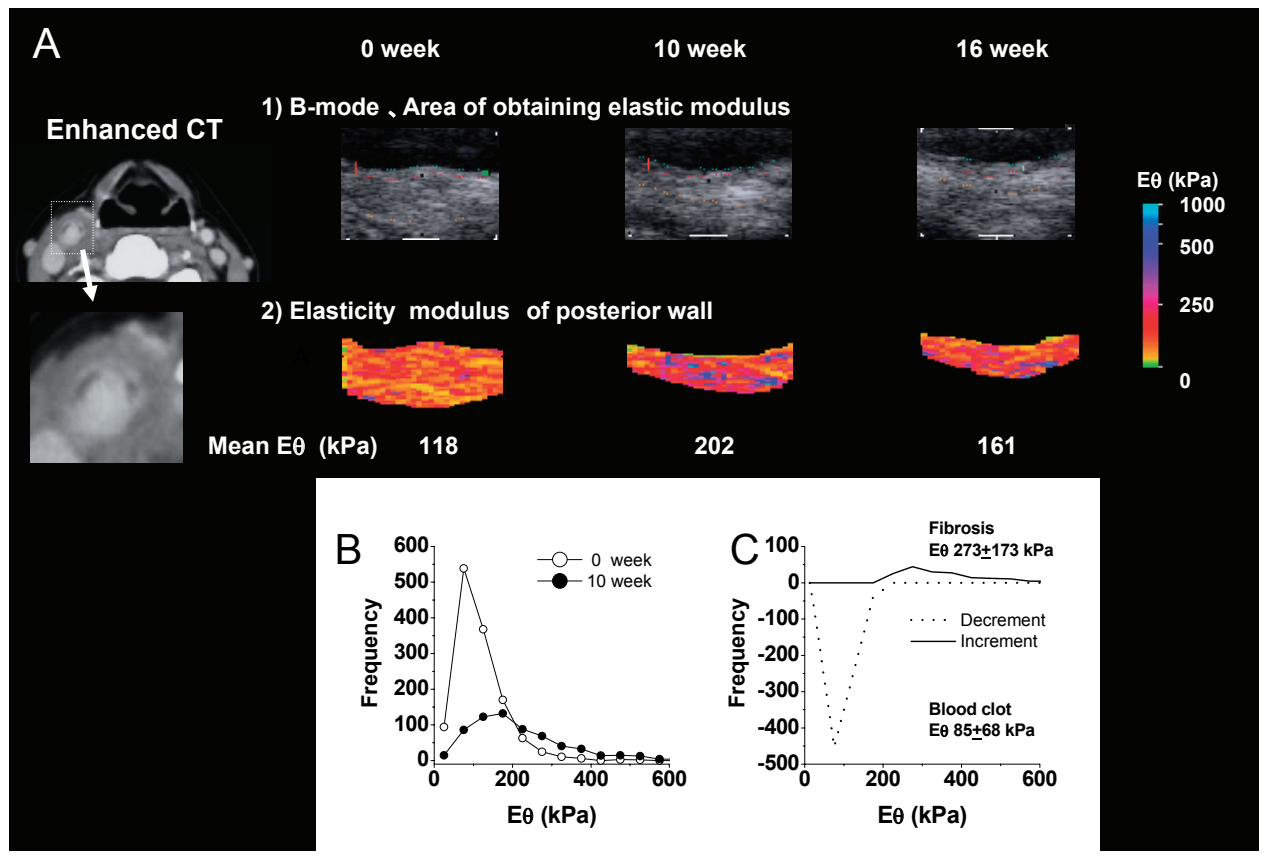

Fig. 3. Aneurysm of right cervical artery in a 43-year-old woman: time course of changes of thrombosis in the pseudolumen.

Computed tomography at 0 weeks showed dissection, and the B-mode and elastic modulus of the lesion in the upper panel at 0 week showed relatively soft tissue elements; however, $E_{\theta}$ values rose over time, indicating the disappearance of the blood clot. In the lower panel (B), histograms of $E_{\theta}$ at 0 weeks and 10 weeks are shown.

0.05). No significant changes in blood pressure or pulse rate were seen (data not shown). Fluvastatin decreased the mean IMT and mean $E_{\theta}$ significantly in the analysis of all sites of all patients (Fig. 6A, ANOVA, $p<0.05)$. Mean $E_{\theta}$ decreased significantly from 3 to 12 months of administration, while mean IMT decreased only after 12 months of administration, compared with control values at 0 months. On the other hand, no change of mean IMT or mean $E_{\theta}$ between 0 and 12 months was seen in the control group of hypercholesterolemia (Fig. 6A, $n=10$ ).

Since it has been reported that native Japanese reach an IMT of $1.1 \mathrm{~mm}$ at age 70 and subjects with max IMT $<1.1 \mathrm{~mm}$ are often classified as not having atherosclerosis ${ }^{5}$, we divided all analyzed sites $(n=242)$ into two groups with a max IMT cut off value of 1.1 $\mathrm{mm}$. In the group with $\max$ IMT $\geq 1.1 \mathrm{~mm}$ (sites: $n=144$ ), both the mean $E_{\theta}$ and mean IMT decreased significantly (Fig. 6B, ANOVA). In comparison with the values at 0 months, the values of mean $E_{\theta}$ at 6 and 12 months and mean IMT at 12 months were significantly decreased. In the group with max IMT $<1.1$ mm (sites: $n=98$ ), the mean $E_{\theta}$ significantly decreased and IMT showed a tendency to decrease (Fig. 6C).

Next, we divided all sites into three groups by

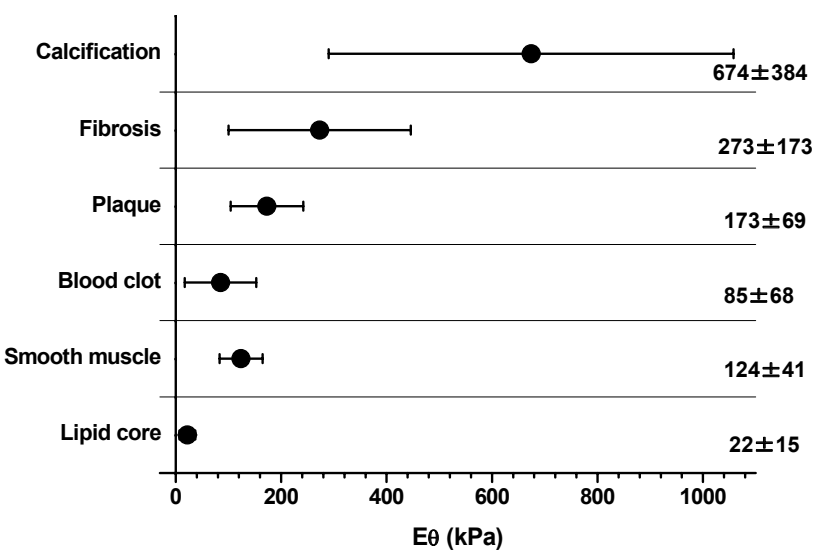

Fig. 4. Elasticity library for $E_{\theta}(\mathrm{kPa})$.

Data of the elasticity library of $E_{\theta}$ by transcutaneous ultrasound were obtained from the observation of various tissues: lipid core, 22 $\pm 15 \mathrm{kPa}$; calcification, $674 \pm 384 \mathrm{kPa}$; lipid core- and calcificationfree plaques, $173 \pm 69 \mathrm{kPa}$; smooth muscle, $104 \pm 32 \mathrm{kPa}$; blood clot, $85 \pm 68 \mathrm{kPa}$; fibrosis, $273 \pm 173 \mathrm{kPa}$. The dark central region of soft plaques was considered to be the lipid core. In healthy subjects free from vascular risk factors, we considered the normal carotid intima-media complex to be smooth muscle. The white regions, which are not accompanied by acoustic shadow, were considered to be calcification regions. Elastic moduli, equivalent to blood clot and fibrosis, were obtained from the observation of an uncomplicated healing process of carotid artery dissection, as shown in Fig. 3. 

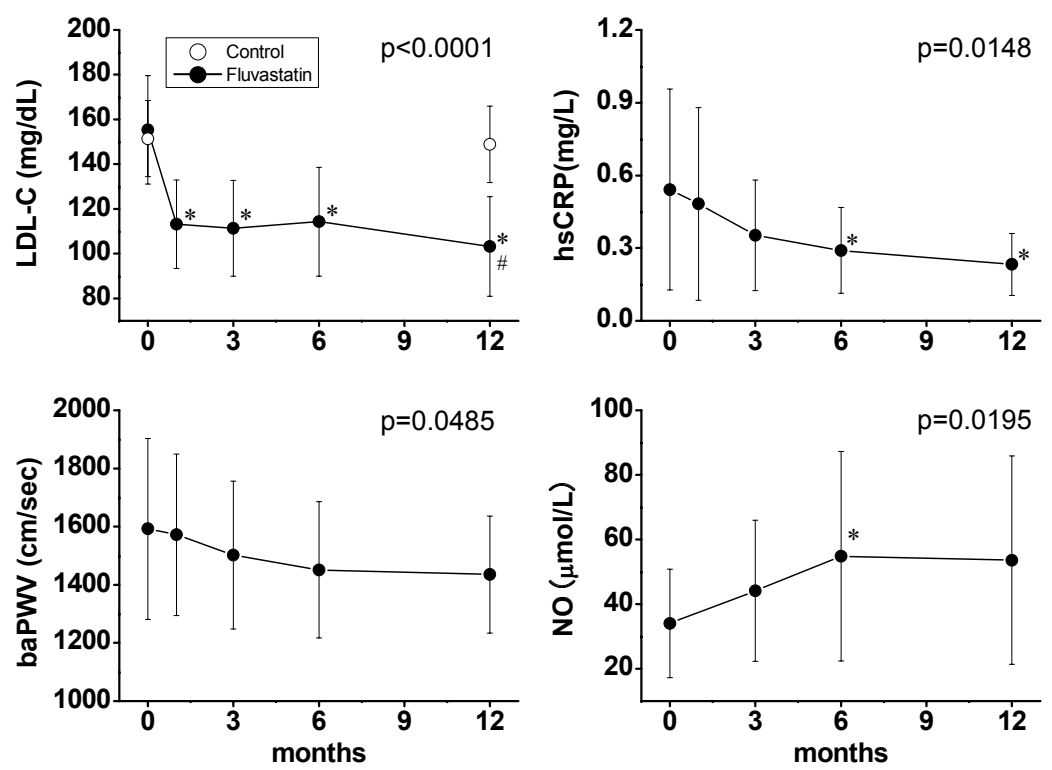

Fig. 5. Effects of fluvastatin on LDL-C, hs-CRP, baPWV and NOx.

A reduction in LDL-C and decreases in baPWV and hs-CRP were noted, along with a gradual increase in NOx. No difference of LDL-C was seen between the control $(n=10)$ and fluvastatin group $(n=62)$ at 0 months. Fluvastatin significantly reduced LDL-C in the comparison of the two groups at 12 months. $P$-values were obtained by ANOVA for the fluvastatin group. ${ }^{*} p<0.05$ vs. 0 months of the fluvastatin group; ${ }^{\#} p<0.05$ vs. 12 months of the control.

the mean $E_{\theta}$ values at 0 months (before administration of fluvastatin), $E_{\theta} \leq 100 \mathrm{kPa}, 100 \mathrm{kPa}<E_{\theta}<200$ $\mathrm{kPa}$, and $200 \mathrm{kPa} \leq E_{\theta}$. In the three groups, IMT did not change and showed a tendency to decrease (Fig. 7). On the other hand, in the group with $E_{\theta} \leq 100 \mathrm{kPa}$, the mean $E_{\theta}$ significantly increased from 1 month after administration of the drug (Fig.7A, sites: $n=42$ ). In the group of $200 \leq E_{\theta}$, the mean $E_{\theta}$ significantly decreased from 1 month after administration of the drug (Fig. 7C, sites: $n=46$ ). In the group of $100 \mathrm{kPa}$ $<E_{\theta}<200 \mathrm{kPa}$, the mean $E_{\theta}$ significantly decreased (Fig. 7B, sites: $n=154$ ).

Two typical examples are shown in Fig. 8, 9. In the advanced thick plaque ( $\max$ IMT $\geq 1.1 \mathrm{~mm}$ and $E_{\theta} \leq 100 \mathrm{kPa}$ ) shown in Fig. 8, fluvastatin decreased IMT and increased $E_{\theta}$ at month 12, particularly in the hardened areas in the superficial layer. Histogram analysis of the data revealed a decrease in areas corresponding to $E_{\theta}$ of $20-200 \mathrm{kPa}$ and an increase in the relatively hardened areas of $E_{\theta}>250 \mathrm{kPa}$ for subgroups showing increased $E_{\theta}$, thought to represent a decrease of lipid/smooth muscle-rich tissue and/or an increase of collagen fibers (based on the elasticity library and Fig. 8).

In the early lesion $(\max I M T<1.1 \mathrm{~mm})$ that showed heterogeneous distribution of $E_{\theta}\left(E_{\theta} \geq 200\right.$
$\mathrm{kPa})$ in the control group, the administration of fluvastatin produced no changes in IMT but resulted in decreased $E_{\theta}$ (Fig. 9). Histogram analysis of the data revealed an increase in areas corresponding to $E_{\theta}$ of 20-200 kPa, as well as a decrease in relatively hardened areas.

\section{Discussion}

We first obtained an in vivo elasticity library with transcutaneous ultrasound from observation of various tissues of the carotid arterial wall by the phased tracking method (Fig.4). In in vitro experiments, as reported previously, the main components of tissue have their own elasticities ${ }^{16)}$. By referring to the elasticity library, we can speculate as to the tissue type whose elastic modulus is close to that of each pixel.

Second, this was a new challenge to show the detection of the effects of fluvastatin on the elasticity of the carotid artery (mean $E_{\theta}$ ). The mean $E_{\theta}$ can be changed by statin treatment; that is, in the group with max $\mathrm{IMT} \geq 1.1 \mathrm{~mm}$, both the mean $E_{\theta}$ and mean IMT decreased significantly. On the other hand, in the max IMT $<1.1 \mathrm{~mm}$ group, the mean $E_{\theta}$ but not the mean IMT decreased significantly.

$\mathrm{B}$-mode ultrasound is widely used to measure 


\section{A All sites (242 sites)}
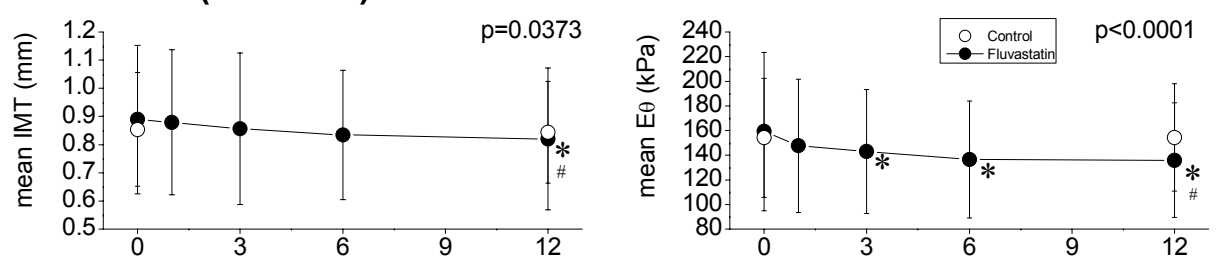

\section{B Max IMT $\geq 1.1 \mathrm{~mm}$ (144 sites)}
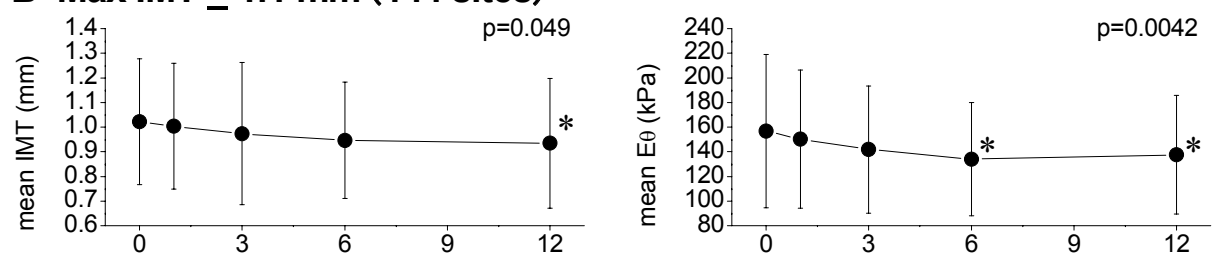

\section{Max IMT < $1.1 \mathrm{~mm}$ (98 sites)}
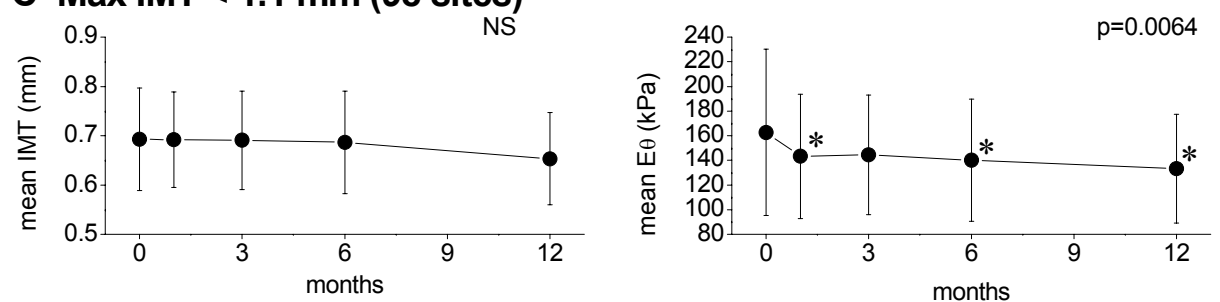

Fig. 6. Effects of fluvastatin on the mean IMT and mean $E_{\theta}$.

(A) At all sites of all patients (242 sites), both the mean IMT and mean $E_{\theta}$ were significantly decreased (ANOVA). Compared with the values at 0 months, the values of mean $E_{\theta}$ at 3,6 and 12 months and mean IMT at 12 month were significantly decreased. No difference of the mean IMT or mean $E_{\theta}$ was seen between the control ( 40 sites in 10) and the fluvastatin group at 0 months. Fluvastatin significantly reduced both the mean IMT and mean $E_{\theta}$ in the comparison of the two groups at 12 months. (B) In the group with max IMT $\geq 1.1 \mathrm{~mm}$ (144 sites), both mean IMT and mean $E_{\theta}$ were significantly decreased. (C) In the group with max IMT $<1.1 \mathrm{~mm}$ (98 sites), mean $E_{\theta}$ but not mean IMT was significantly decreased. $P$-values were obtained by ANOVA for the fluvastatin group. ${ }^{*} p<0.05$ vs. 0 months of the fluvastatin group; ${ }^{*} p<0.05$ vs. 12 months of the control.

carotid IMT, an early change that precedes the development of macroscopic plaques. Carotid IMT is increasingly being used for cardiovascular risk stratification and as an intermediate end point in clinical tri$\mathrm{als}^{19)}$, but it has limited ability to determine the extent of plaque burden and the nature of plaque composition. IBS analysis has shown associations between echo-lucent carotid plaques and lower HDL-C cholesterol, elevated triglycerides, elevated blood pressure and elevated blood sugar levels ${ }^{7,8}$; however, the IBS index simply reflects differences in echogenicity, but does not show the true elasticity of the arterial wall. Carotid stiffness, considered an index of sclerosis, can be determined by the following equation: $\beta=\ln (\mathrm{SBP} /$ $\mathrm{DBP}) /[(\mathrm{Ds}-\mathrm{Dd}) / \mathrm{Dd}]$, where Ds and Dd are the endsystolic and end-diastolic diameters of the common carotid artery, respectively ${ }^{20,21)}$; however, this value does not indicate the two-dimensional distribution of carotid arterial wall elasticity. Under the assumption of a blood vessel is a uniform cylinder pipe, PWV and stiffness $\beta$ can be used to evaluate average elastic properties of the whole transmission passage and the entire circumference by measuring pulse wave velocity between dozens of centimeters and alteration of the diameter by beat, respectively. These methods are not necessarily suitable for comparing plaques or individuals, or for assessing qualitative changes. In contrast, our method enables the measurement of elasticity distribution in the intima-medial region by ultrasound and the evaluation of local (lesion) elastic properties by measuring mural thickness change. In a previous study, age, blood pressure and pulse rate were shown to be independent determinants of the average values $E_{\theta}$ of elasticity distribution ${ }^{15)}$.

In the present study, an elasticity library with transcutaneous ultrasound was obtained by observing various tissues of the carotid arterial wall (Fig. 4). We were fortunate to be able to observe an uncomplicated 
A $\mathrm{E}_{\theta} \leq 100(\mathrm{kPa})$ at 0 months (42 sites)
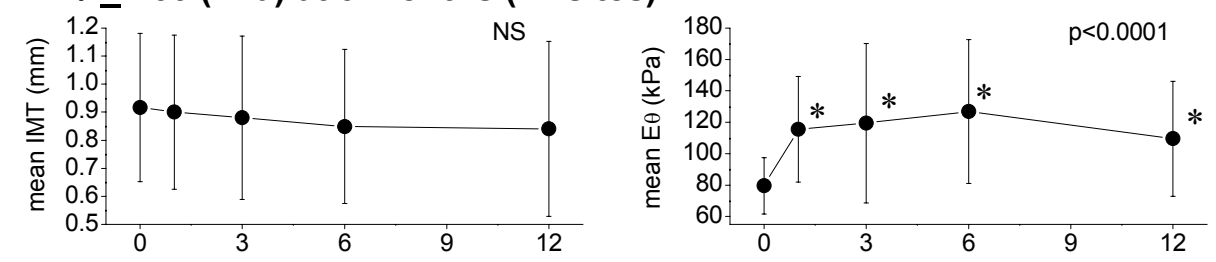

\section{B $100<\mathrm{E} \theta<200(\mathrm{kPa})$ at 0 months (154 sites)}
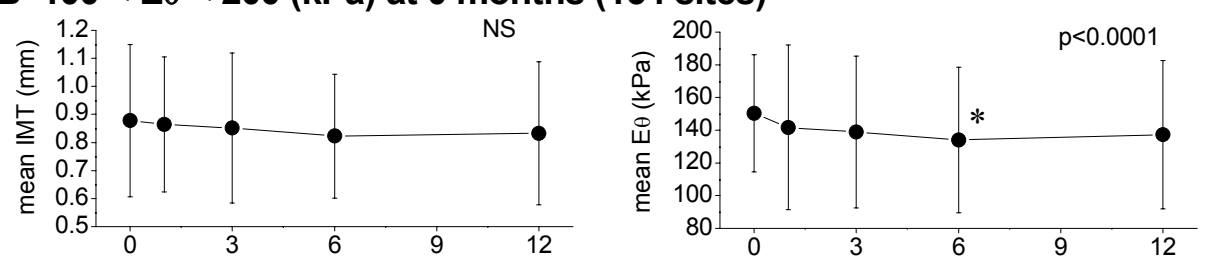

C $\mathrm{E} \theta \geq 200(\mathrm{kPa})$ at 0 months (46 sites)
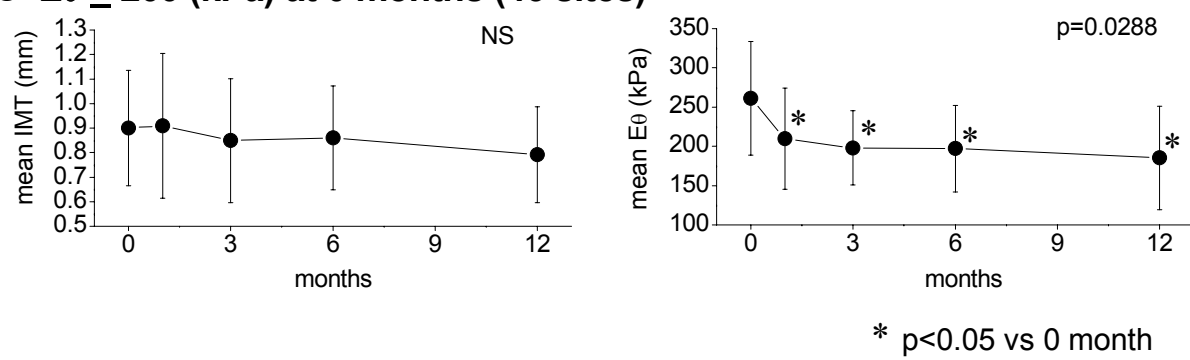

Fig.7. Effects of fluvastatin on the mean IMT and mean $E_{\theta}$ in the three groups according to the mean $E_{\theta}$ values at 0 months.

(A) In the group with $E_{\theta} \leq 100$ (42 sites), the mean $E_{\theta}$ was significantly increased after 1-month administration of the drug. (B) In the group with $100 \mathrm{kPa}<E_{\theta}<200 \mathrm{kPa}$ (154 sites), mean $E_{\theta}$ was significantly decreased. (C) In the group with $200 \leq E_{\theta}$ (46 sites), the mean $E_{\theta}$ was significantly decreased after 1 -month administration of the drug.

healing process following carotid artery dissection, since such cases usually heal spontaneously ${ }^{22)}$, and to obtain the elastic modulus, equivalent to blood clot and fibrosis information by histogram. When the relative interrelationships for elasticity in the corresponding vascular tissues were considered in this study, a remarkable similarity was noted to the elasticity findings for lipids, thrombus, smooth muscle, fibrotic tissue and calcification in isolated atherosclerotic vascular specimens as documented by Inagaki et al. ${ }^{23)}$.

Many risk factors are associated with endothelial dysfunction, including hypercholesterolemia, hypertension, cigarette smoking and diabetes mellitus. Increasing evidence indicates that oxidized low-density lipoprotein cholesterol (ox-LDL) plays an important role in endothelial dysfunction. Ox-LDL induces endothelial injury, inhibits apoptosis, monocyte adhesion and platelet aggregation, and also inhibits the expression/activity of endothelial nitric oxide synthase $(\mathrm{eNOS})^{24)}$. Previous research has shown that 3-hydroxy3-methylglutaryl coenzyme A reductase inhibitors (statins) improve endothelial function through mechanisms that go beyond their primary therapeutic effects ${ }^{25-28)}$. Alteration of the endothelial function might result from increases in eNOS activity, reduced production of free radicals, inhibition of ox-LDL action, or other undefined mechanisms. In addition, coronary spasm is associated with endothelial dysfunction, and fluvastatin has been reported to reduce the number of patients with ACh-induced coronary spasm, which is associated with endothelial dysfunction, in comparison with conventional calcium channel blocker therapy ${ }^{26)}$. In our study, fluvastatin increased NO and decreased hs-CRP. In the group with max IMT $<1.1 \mathrm{~mm}$, considered to be in the range of subclinical atherosclerotic findings, the elastic modulus was significantly reduced. This may be related to fluvastatin-induced improvement in endothelial dysfunction. Additionally, statin treatment is reported to decrease carotid arterial stiffness but not IMT (initial max IMT $0.95 \pm 0.4 \mathrm{~mm}$ ) in patients with hypercholesterolemia ${ }^{20)}$. Ultrasonic strain imaging has the 


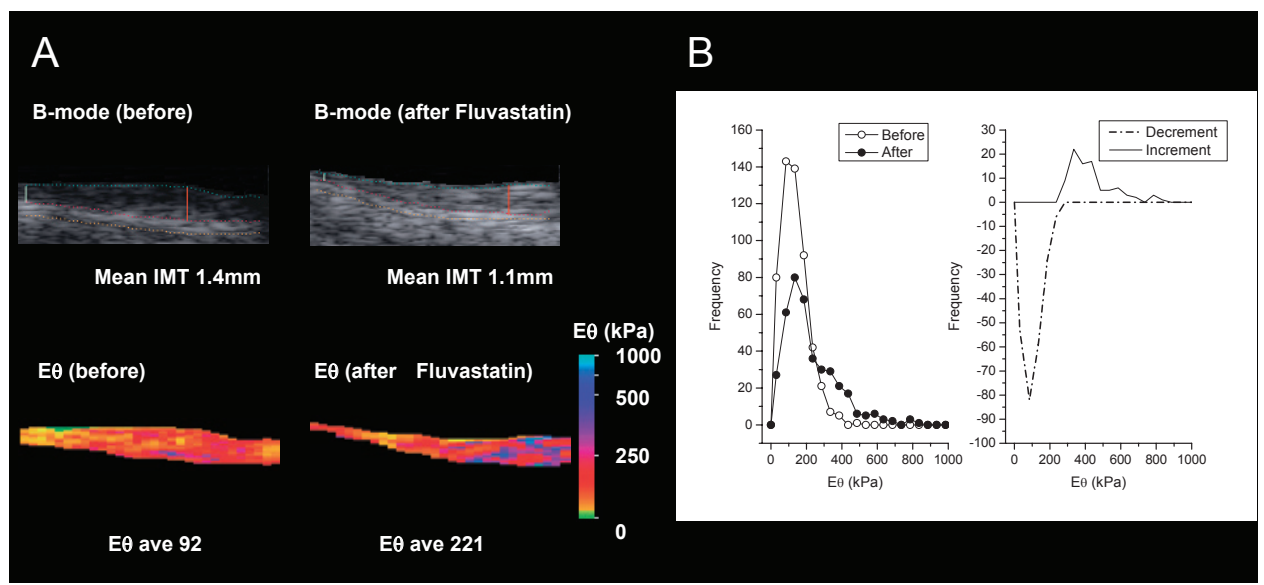

Fig. 8. A case of increased mean $E_{\theta}$ after fluvastatin administration (50-year-old man).

Images before and after administration of the drug for 12 months are shown. In the histograms, the increase in $E_{\theta}$ showed a decrease of lipid/smooth muscle-rich tissue and/or an increase of collagen fibers, deterring B-mode findings.

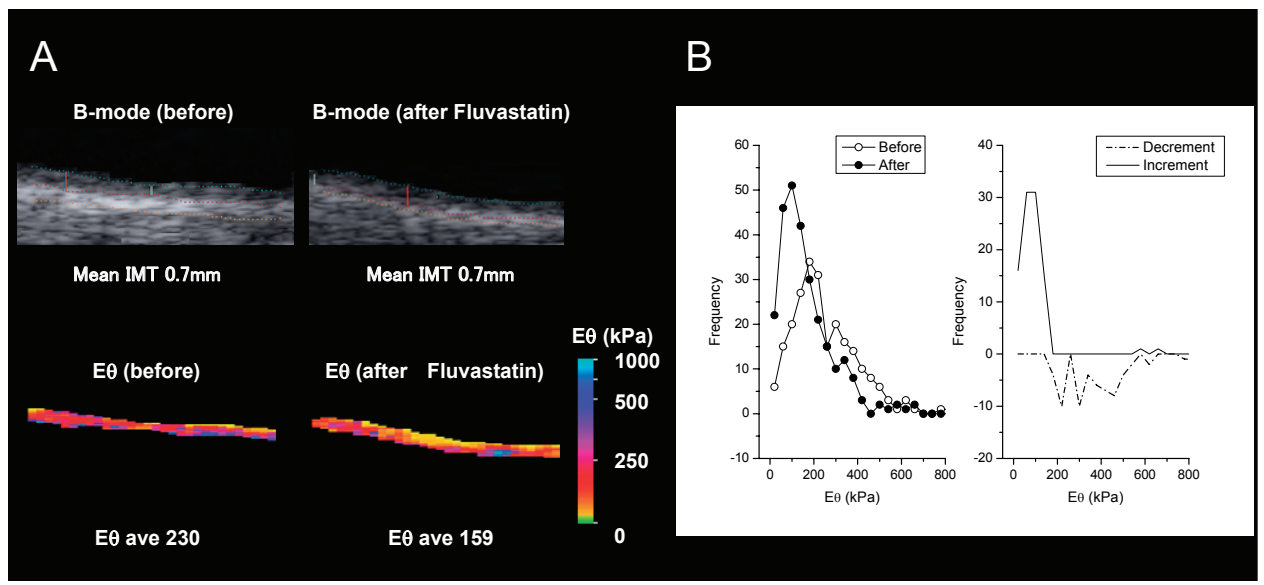

Fig. 9. A case of decrease in mean $E_{\theta}$ after fluvastatin administration (50-year-old man).

Images before and after administration of the drug for 12 months are shown. In the histograms, the decrease in $E_{\theta}$ shows an increase in areas corresponding to $E_{\theta}$ of $20-200 \mathrm{kPa}$, as well as a decrease in relatively hardened areas, indicating improvement of endothelial dysfunction.

potential to become a sensitive tool for detecting the effects of early statin intervention.

Recent studies, including those on advanced plaque formation, have also shown benefits from intensive statin therapy in slowing and even reversing the progression of atherosclerosis, as assessed by surrogate imaging measures such as IVUS and carotid IMT. In a study of the effectiveness of rosuvastatin against increases of intima media thickness, a statistically significant slowing in the progression rate of maximum carotid IMT (initial max IMT $1.15 \pm 0.19 \mathrm{~mm}$ ) was observed in the rosuvastatin group relative to the placebo group for 12 carotid sites, as well as for each individual segment ${ }^{27)}$. Subanalysis of this METEOR study found that in the rosuvastatin group, a greater trend toward regression was seen in patients with more risk factors and greater baseline thickness ${ }^{28)}$. Similarly, in our study, a greater trend toward regression was seen in the advanced plaque group $(\max I M T \geq 1.1$ $\mathrm{mm}$, Fig. 6).

As shown in Fig. 7, changes in mean $E_{\theta}$ may be related to an incremental beneficial effect on plaque stabilization with statins; for example, in the group with $E_{\theta} \leq 100 \mathrm{kPa}$, the softer areas, mean $E_{\theta}$ significantly increased from 1 month after administration of the drug. This may be the result of a diminution of 
the lipid-rich core, a reduction of inflammatory cells within the plaques, decreased macrophage activation as well as foam cell formation, and events related to thickening of the fibrous cap as reported by several investigators ${ }^{29-31)}$. On the other hand, in the group with $200 \mathrm{kPa} \leq E_{\theta}$, the harder areas, mean $E_{\theta}$ was significantly decreased. This may be related to improvement of endothelial dysfunction, such as paradoxic vasoconstriction of cardiac vessels. In the group with $100 \mathrm{kPa}<E_{\theta}<200 \mathrm{kPa}$, the middle range of the elastic modulus, mean $E_{\theta}$ significantly decreased. This may be the result of variable changes of plaque components mentioned above.

On the basis of comparing histograms for tissue characterization (Fig.4) and fluvastatin-induced changes in the intima-media complex (Fig. 6, 7, 8, 9), it may be possible to perform tissue characterization by comparing elasticity distribution and chronological changes in the distribution as measured by ultrasound. Collagen and smooth-muscle content have been reported to be important factors in determining the stability of the fibrous cap on atherosclerotic plaques; therefore, correlations between elasticity and tissue elements within the arterial wall may provide useful information for the noninvasive diagnosis of plaque vulnerability ${ }^{23)}$.

The present study has the following limitations: Although plaque can be evaluated three-dimensionally and relatively more easily with certain methods, such as computed tomography and magnetic resonance imaging, conventional IMT measurement is performed in one cross-section, making it difficult to construct three-dimensional images, which would be a future hurdle to overcome. In addition, measurement of one cross-section influences not only elasticity, but also the IMT. In the present study, to measure the same sites of carotid arteries, one well-trained sonographer performed the IMT and elastic modulus measurements by comparing current B-mode images with past Bmode images on a $\mathrm{PC}$ and positioned the probe so as to measure a similar position. Finally, the spatial averages of the elastic moduli were compared so that the mean value would be the same when the same area was included.

Plaques consist of various tissue components, and we previously reported the comparison of the elastic modulus with histologically corresponding tissues, such as lipids, blood clots and calcification ${ }^{16}$. In that report, the distributions of lipids and blood clots were relatively narrow and pure; however, those of collagen, smooth muscle and calcification were wide and rather scattered. The rank of the center of elasticity distributions obtained in the present study was similar to that of in vitro experiments, indicating that it is impossible to obtain completely pure plaque tissue components, although the major components of the plaque can be detected and a classification can be made based on the elastic modulus.

$E_{\theta}$ may be an ideal marker to show the elasticity of the arterial wall itself because $E \mathrm{r}$ (apparent elasticity) is influenced not only by an increase in elasticity but also by the increasing thickness of the arterial wall at the same location, especially in areas of relatively great thickness; however, the influence of blood pressure on the library cannot be neglected. The effects of aging on the mean IMT and mean $E_{\theta}$ are shown in Fig. 1. It seems that $E_{\theta}$ does not increase greatly in normotensives, even with advancing age. In hypertensives, however, $E_{\theta}$ was found to increase not only with decrements of strain (the denominator of Eq. (3) for elastic modulus) via tissue characteristics such as fibrosis, calcification and local contraction of smooth muscle, but also with increments of pulse pressure (the numerator of Eq. (3) for elastic modulus). A comparison with pathological findings for the elastic modulus is needed; however, in humans it is ethically unacceptable to compare ultrasonic images with pathological findings in early-stage carotid arterial atherosclerosis. In addition, an elastic modulus obtained by means of transcutaneous ultrasound differs from that obtained from isolated atherosclerotic vascular specimens, since the former is also affected by subcutaneous tissue, such as muscle and fat.

In summary, the present non-invasive echographic carotid arterial elasticity data suggest that this method is useful for classification and evaluation of atherosclerotic plaques that increase with progressive lipid deposition and that the measurement data may reflect chronological and histopathological changes.

\section{References}

1) Pignoli P, Tremoli E, Poli A, Oreste P, Paoletti R: Intimal plus medial thickness of the arterial wall; a direct measurement with ultrasound imaging. Circulation, 1986; 74 : 1399-1406

2) Lee RT, Grodzinsky AJ, Frank EH, Kamm RD, Schoen FJ: Structure-dependent dynamic mechanical behavior of fibrous caps from human atherosclerotic plaques. Circulation, 1991; 83: 1764-1770

3) Howard G, Sharrett AR, Heiss G, Evans GW, Chambless LE, Riley WA, Burke GL: Carotid artery intimal medial thickness distribution in general populations as evaluated by B-mode ultrasound. ARIC Investigators. Stroke, 1993; 24: 1297-1304

4) Simon A, Gariepy J, Chironi G, Megnien, Levenson: Intima-media thickness: a new tool for diagnosis and treatment of cardiovascular risk. J Hypertens, 2002; 20: 
159-169

5) Watanabe H, Yamane K, Fujikawa R, Okubo M, Egusa G, Kohno N: Westernization of lifestyle markedly increases carotid intima-media wall thickness (IMT) in Japanese people. Atherosclerosis, 2003; 166: 67-72

6) Salonen JT, Salonen R: Ultrasound B-mode imaging in observational studies of atherosclerotic progression. Circulation, 1993; 87 (3 Suppl): II 56-65

7) Takiuchi S, Rakugi H, Honda K, Masuyama T, Hirata N, Ito H, Sugimoto K, Yanagitani Y, Moriguchi K, Okamura A, Higaki J, Ogihara T: Quantitative ultrasonic tissue characterization can identify high-risk atherosclerotic alteration in human carotid arteries. Circulation, 2000; 102: 766-770

8) Yamagami H, Kitagawa K, Nagai Y, Hougaku H, Sakaguchi M, Kuwabara K, Kondo K, Masuyama T, Matsumoto M, Hori M: Higher levels of interleukin-6 are associated with lower echogenicity of carotid artery plaques. Stroke, 2004; 35: 677-681

9) Kanai H, Sato M, Koiwa Y: Transcutaneous measurement and spectrum analysis of heart wall vibrations. IEEE Trans Ultrasonics Ferroelect Freq Control, 1996; 43: 791-810

10) Hasegawa $H$, Kanai $H$, Hoshimiya $N$ : Evaluating the regional elastic modulus of a cylindrical shell with nonuniform wall thickness. J Med Ultrasonics, 2004; 31: 8190

11) Kanai H, Watanabe $M$, Hasegawa $H$ : Carotid artery intima-media elasticity measured by ultrasonography in subjects with normal intima-media thickness correlates well with atherosclerosis risk factors. Circulation, 2002; 106: II-442

12) Kanai H, Hasegawa H, Ichiki M, Tezuka F, Koiwa Y: Elasticity imaging of atheroma with transcutaneous ultrasound: preliminary study. Circulation, 2003; 107: 30183021

13) Yamagishi T, Kato M, Koiwa Y: Usefulness of measurement of carotid arterial wall elasticity distribution in detection of early-stage atherosclerotic lesions caused by cigarette smoking. J Med Ultrasonics, 2006; 33: 203-210

14) Hasegawa $H$, Kanai $H$, Ichiki $M$ : Tissue structure of arterial wall revealed with elasticity imaging. J Med Ultrasonics, 2007; 34: 73-74

15) Yamagishi T, Kato M, Koiwa Y, Hasegawa H, Kanai H: Impact of lifestyle-related diseases on the carotid arterial wall elasticity as evaluated by an ultrasonic phased-tracking method in Japanese subjects (submitted to J Atheroscler Thromb)

16) Inagaki J, Hasegawa $H$, Kanai M: Construction of reference data for tissue characterization of arterial wall based on elasticity image. Jpn J Appl phys, 2005; 44: 4593-4597

17) Munakata M, Ito N, Nunokawa T, Yoshinaga K: Utility of automated brachial ankle pulse wave velocity measurements in hypertensive patients. Am J Hypertens, 2003; 16: 653-657

18) Green LC, Wagner DA, Glogowski J, Skipper PL, Wishnok JS, Tannenbaum SR: Analysis of nitrate, nitrite and $[15 \mathrm{~N}]$ nitrate in biological fluids. Anal Biochem 1982; 126: $131-138$

19) Roberts WL, Sedrick R, Moulton L, Spencer A, Rifai N:
Evaluation of four automated high-sensitivity C-reactive protein methods: implications for clinical and epidemiological applications. Clin Chem, 2000; 46: 461-468

20) Davidson MH: Overview of prevention and treatment of atherosclerosis with lipid-altering therapy for pharmacy directors. Am J Manag Care, 2007; 13 Suppl 10: S260-269

21) Saba PS, Roman MJ, Longhini C, Scorzoni D, Pini R, Devereux RB, Ganau A: Carotid intimal-medial thickness and stiffness are not affected by hypercholesterolemia in uncomplicated essential hypertension. Arterioscler Thromb Vasc Biol, 1999; 19: 2788-2794

22) Mizuguchi $Y$, Oishi $Y$, Miyoshi $H$, Iuchi $A$, Nagase $N$, Oki T: Impact of statin therapy on left ventricular function and carotid arterial stiffness in patients with hypercholesterolemia. Circ J, 2008; 72: 538-554

23) Schievink IW: Spontaneous Dissection of the Carotid and Vertebral Arteries. N Engl J Med, 2001; 344: 898-906

24) Li D, Mehta JL: 3-hydroxy-3-methylglutaryl coenzyme A reductase inhibitors protect against oxidized low-density lipoprotein-induced endothelial dysfunction. Endothelium, 2003; 10: 17-21

25) Boodhwani M, Nakai Y, Voisine P: High-dose atorvastatin improves hypercholesterolemic coronary endothelial dysfunction without improving the angiogenic response. Circulation, 2006; 114(Suppl 1): I 402-408

26) Yasue $H$, Mizuno $Y$, Harada E, Itoh $T$, Nakagawa $H$, Nakayama M, Ogawa H, Tayama S, Honda T, Hokimoto S, Ohshima S, Hokamura Y, Kugiyama K, Horie M, Yoshimura M, Harada M, Uemura S, Saito Y; SCAST (Statin and Coronary Artery Spasm Trial) Investigators: Effects of a 3- hydroxy-3-methylglutaryl coenzyme A reductase inhibitor, fluvastatin, on coronary spasm after withdrawal of calcium-channel blockers. J Am Coll Cardiol, 2008; 51: 1742-1748

27) Crouse JR, Raichlen JS, Riley WA, Evans GW, Palmer MK, O'Leary DH, Grobbee DE, Bots ML; METEOR Study Group: Effect of rosuvastatin on progression of carotid intima-media thickness in low-risk individuals with subclinical atherosclerosis. The METEOR trial. JAMA, 2007; 297: 1344-1353

28) Evans GW, Raichlen JS, Bots ML: on behalf of the METEOR Study Group. Rosuvastatin treatment in carotid atherosclerosis: effect of Framingham risk factors and baseline intima-media thickness in the METEOR trial. Circulation, 2007; 116(suppl II): 848. Abstract 3730

29) Yano T, Kawano H, Mochizuki H, Doi O, Nakamura T, Saito Y: Atherosclerotic plaques composed of a large core of foam cells covered with thin fibrous caps in twiceinjured carotid arterial specimens obtained from high cholesterol diet-fed rabbits. J Atheroscler Thromb, 2000; 7: 83-90

30) Suzuki H, Kobayashi H, Sato F, Yonemitsu Y, Nakashima Y, Sueishi K: Atherosclerosis and extracellular matrix. J Atheroscler Thromb, 2003; 10: 109-116

31) Suzuki H, Kobayashi H, Sato F, Yonemitsu Y, Nakashima Y, Sueishi K: Plaque-stabilizing effect of pitavastatin in Watanabe heritable hyperlipidemic (WHHL) rabbits. J Atheroscler Thromb, 2003; 10: 109-116 